\title{
Schulterschluss für eine rasche Wiedereingliederung
}

\section{Serkan Isik ${ }^{a}$, Pia Wälti ${ }^{b}$}

a Unternehmenskommunikation Suva; ${ }^{b}$ Leiterin Kommunikation, IV-Stelle Solothurn

Wirtschaftsverbände, Ärzteschaften sowie die Sozialversicherer IV-Stelle Solothurn und Suva des Kantons Solothurn setzen sich gemeinsam dafür ein, dass arbeitsunfähige Menschen möglichst rasch an ihren Arbeitsplatz zurückkehren können. Für eine effiziente Umsetzung haben sie an einem runden Tisch Grundsätze erarbeitet und sich mit ihrer Unterschrift dazu verpflichtet, diese bei der Zusammenarbeit einzuhalten.

Studien belegen, dass die Eingliederungschancen nach sechsmonatiger Arbeitsunfähigkeit um rund 50 Prozent abnehmen. Deshalb ist es entscheidend, dass sich das soziale und das berufliche Umfeld der Betroffenen so früh wie möglich dafür einsetzen, dass arbeitsunfähige Personen so rasch wie möglich wieder an den Arbeitsplatz zurückkehren können. Durch eine koordinierte Zusammenarbeit zwischen dem Kantonal-Solothurnischen Gewerbeverband, der Solothurner Handelskammer, den Hausärzten Solothurn (HASO) und der Gesellschaft der Ärztinnen und Ärzte des Kantons Solothurn (GAeSO) sowie den Sozialversicherern IV-Stelle Solothurn und Suva Solothurn sollen Arbeitsausfälle und Gesundheitskosten reduziert werden. Die Projektbeteiligten haben am 4. Februar eine verbindliche Zusam- menarbeitsvereinbarung unterschrieben, die in dieser Form schweizweit einzigartig ist und den Kanton Solothurn zum Vorreiter macht.

\section{Entscheidend ist die Kommunikation untereinander}

Eine Arbeitsunfähigkeit stellt die Arbeitgeber, die behandelnden Ärzte sowie die zuständige Sozialversicherung vor Herausforderungen. Während der Arbeitgeber möglichst transparente Informationen über die Rückkehr des Verunfallten erwartet, fehlen den behandelnden Ärzten für die Festlegung der Arbeitsunfähigkeit oft die notwendigen Kenntnisse über den Arbeitsplatz des Patienten. «Wir sind davon überzeugt, dass ein ge-

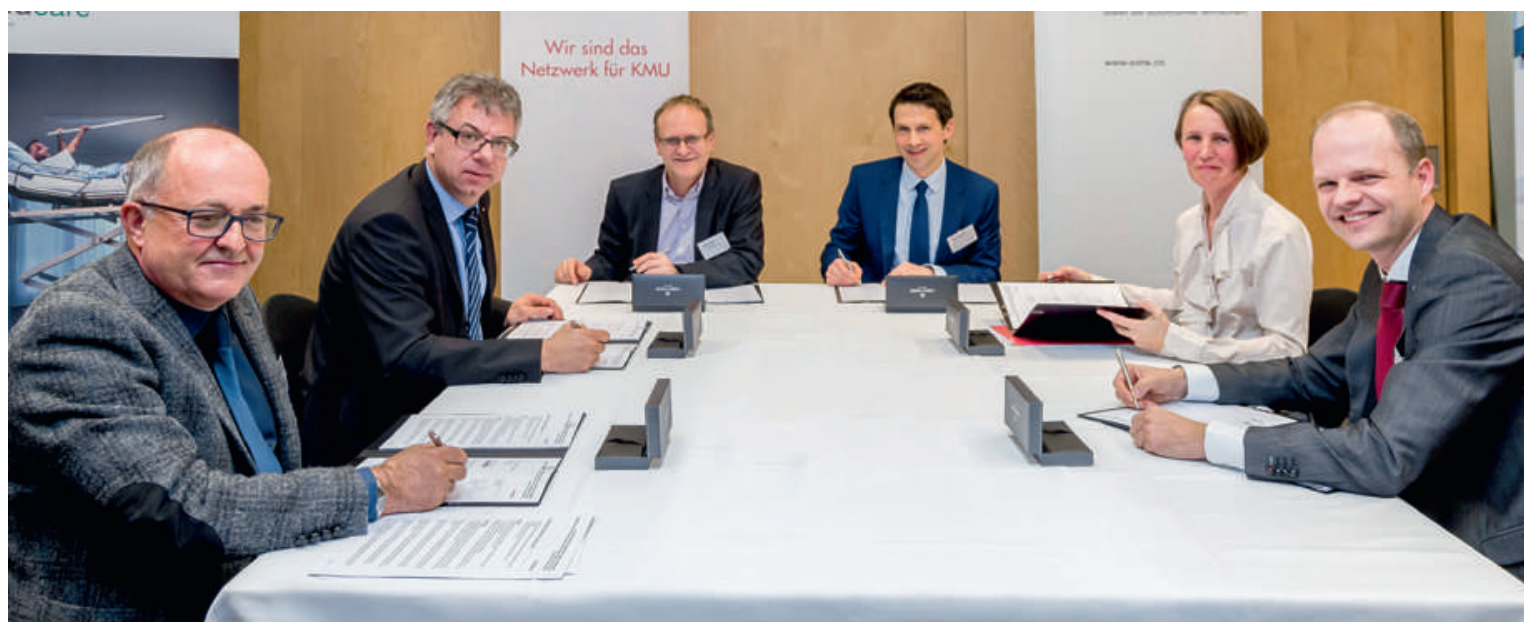

Sie haben die Vereinbarung zur Zusammenarbeit für ihre jeweiligen Organisationen unterschrieben (von links): Lukas Meier, Präsident der Solothurner Ärztegesellschaft, Andreas Gasche, Präsident des kantonalen Gewerbeverbands, Martin Gabl, Geschäftsleiter der IV-Stelle Solothurn, Kilian Bärtschi, Agenturleiter der Suva Solothurn, Gabriela Kissling, Co-Präsidentin der Hausärzte Solothurn, und Daniel Probst, Direktor der Solothurner Handelskammer. 
zielter Dialog zwischen Arbeitgebern und den behandelnden Ärzten die Abwesenheit eines Arbeitnehmenden verkürzen kann", sagt Dr. Gabriela Kissling, Co-Präsidentin Hausärzte Solothurn. Denn die Beurteilung einer Arbeitsfähigkeit setze voraus, dass der Arzt die konkrete Arbeitsplatzsituation des Patienten kenne, über Wiedereingliederungsangebote des Arbeitgebers informiert sei und wisse, welche Schonarbeitsplätze das Unternehmen anbiete. «Arbeitgeber, Ärzte und Sozialversicherungen müssen bei Unklarheiten oder komplexen Sachverhalten aufeinander zugehen. Nur so kann die Wiedereingliederung gefördert werden", sagt Martin Gabl, Geschäftsleiter der IV-Stelle Solothurn. Denn das soziale und berufliche Umfeld entscheide massgeblich über den Verlauf der Wiedereingliederung.

Korrespondenz: Serkan Isik Unternehmenskommunikation Suva

Tel. 0414196514 serkan.isik@suva.ch www.suva.ch

\section{Pia Wälti, Leiterin} Kommunikation IV-Stelle Solothurn Tel. 0326862446 pia.waelti@ivso.ch www.ivso.ch

\section{Grundsätze bilden den Rahmen der Zusammenarbeit}

«Um Informationslücken zu schliessen und die Kommunikation unter den Ärzten, den Arbeitgebern und den Sozialversicherungen zu gewährleisten, haben wir in partnerschaftlicher Zusammenarbeit Grundsätze und ein Merkblatt erarbeitet», sagt Kilian Bärtschi, Agenturleiter der Suva Solothurn. Die Unterlagen thematisieren die Schwerpunkte der Zusammenarbeit. So wird unter anderem im Detail definiert, welche Informationen auf einem Arbeitsunfähigkeitszeugnis vorhanden sein müssen, wie die Arbeitsunfähigkeit in der Praxis festgesetzt wird oder auf welche zusätzlichen Informationen zum Arbeitsunfähigkeitszeugnis der Arbeitgeber Anspruch hat. «Mit unseren Unterschriften verpflichten wir uns alle, die erarbeiteten Grundsätze bei der täglichen Arbeit einzuhalten. Wir erhoffen uns, dass so potentielle Missverständnisse erst gar nicht entstehen und wir es in partnerschaftlicher Zusammenarbeit schaffen werden, Ausfalltage zu reduzieren", sagt Daniel Probst, Direktor der Solothurner Handelskammer.

Bildnachweis zVg Kilian Bärtschi

\section{Merkblatt zur Zusammenarbeit}

Die im Hinblick auf eine Optimierung der Wiedereingliederung zusammenarbeitenden Partner haben ein Merkblatt mit wichtigen Hinweisen zusammengestellt. Es enthält beispielsweise Information zur Festsetzung der Arbeitsunfähigkeit in der Praxis und behandelt Fragen wie die rückwirkende Festsetzung der Arbeitsunfähigkeit oder die Arbeitsunfähigkeit von unbestimmter Dauer. Die vollständige Fassung des Merkblatts finden Sie als PDF unter www.saez.ch $\rightarrow$ Archiv $\rightarrow$ Zeitschriftenarchiv $\rightarrow$ Ausgabe 2016/09 $\rightarrow$ Artikel "Schulterschluss für eine rasche Wiedereingliederung" $\rightarrow$ Merkblatt zur Zusammenarbeit. 